\title{
Optical differential reflectance spectroscopy for photochromic molecules on solid surfaces
}

Cite as: Rev. Sci. Instrum. 89, 033113 (2018); https://doi.org/10.1063/1.5019415

Submitted: 14 December 2017 . Accepted: 01 March 2018 . Published Online: 28 March 2018

Fabian Nickel, Matthias Bernien, Uwe Lipowski, and Wolfgang Kuch (D)
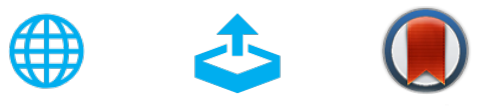

\section{ARTICLES YOU MAY BE INTERESTED IN}

Universal sensor based on the spectroscopy of glow discharge for the detection of traces of atoms or molecules in air

Review of Scientific Instruments 89, 033105 (2018); https://doi.org/10.1063/1.4989904

Laser frequency stabilization using a transfer interferometer

Review of Scientific Instruments 89, 033109 (2018); https://doi.org/10.1063/1.5012000

Dianion diagnostics in DESIREE: High-sensitivity detection of $C_{n}^{2-}$ from a sputter ion source Review of Scientific Instruments 89, 033112 (2018); https://doi.org/10.1063/1.5010077

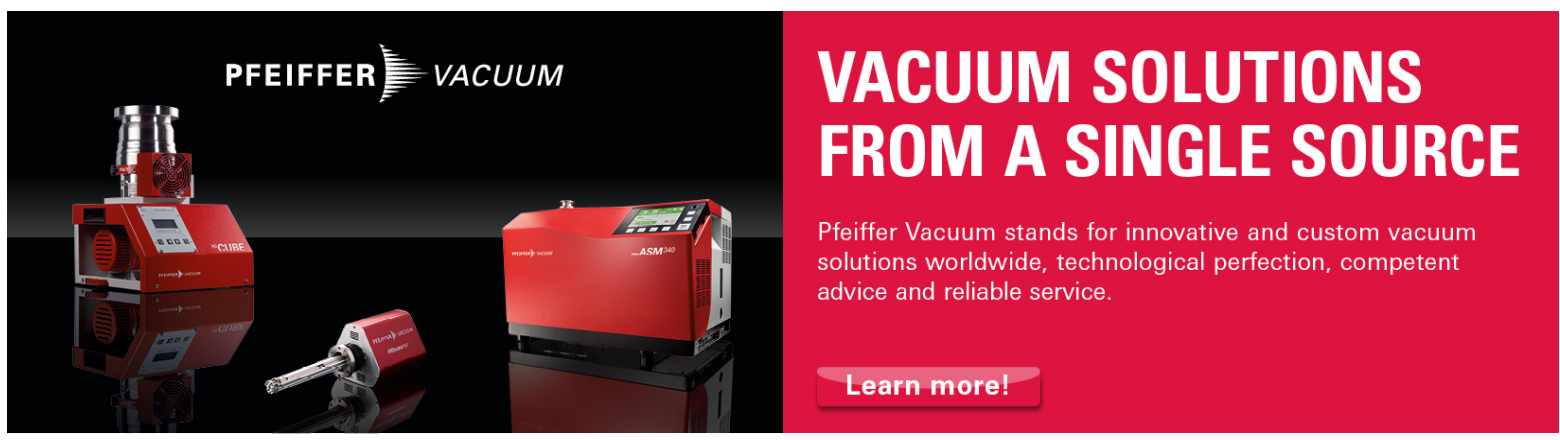




\title{
Optical differential reflectance spectroscopy for photochromic molecules on solid surfaces
}

\author{
Fabian Nickel, ${ }^{\text {a) }}$ Matthias Bernien, Uwe Lipowski, and Wolfgang Kuch \\ Institut für Experimentalphysik, Freie Universität Berlin, Arnimallee 14, 14195 Berlin, Germany
}

(Received 14 December 2017; accepted 1 March 2018; published online 28 March 2018)

\begin{abstract}
Optical reflectance of thin adsorbates on solid surfaces is able to reveal fundamental changes of molecular properties compared to bulk systems. The detection of very small changes in the optical reflectance required several technical improvements in the past decades. We present an experimental setup that is capable of high-quality measurements of submonolayers and ultrathin layers of photochromic molecules on surfaces as well as quantifying their isomerization kinetics. By using photomultipliers as detectors, an enhancement of the signal-to-noise ratio by a factor of three with a total reduction of light exposure on the sample by at least four orders of magnitude is achieved. The potential of the experimental setup is demonstrated by a characterization of the photoswitching and thermal switching of a spirooxazine derivate on a bismuth surface. Published by AIP Publishing. https://doi.org/10.1063/1.5019415
\end{abstract}

\section{INTRODUCTION}

Optical differential reflectance spectroscopy (DRS) compares the reflectance of a clean substrate with one that has been modified. ${ }^{1}$ Possible modifications are, e.g., gas or molecule adsorption. The relative change of the reflectance gives indirect information on the change of the surface properties or, in case of molecules, of the inorganic-organic system. For investigation of adsorbates on surfaces, typically characterization methods such as low-energy electron diffraction (LEED), scanning tunneling microscopy (STM), x-ray absorption spectroscopy (XAS), or x-ray photoelectron spectroscopy (XPS) are used. The main difference to optical methods is the smaller penetration depth, which renders them highly surface sensitive. These methods have certain drawbacks, e.g., electrons and $x$ rays can strongly interact with the adsorbate, which can even lead to a total destruction. STM, on the other hand, only gives local properties and limited information on the chemical structure.

The optical method of DRS has been applied since decades, e.g., for alloys ${ }^{2}$ or the change of semiconductor properties after treating by adsorbates. ${ }^{3}$ More recently, DRS has been used for the investigation of in-situ-grown organic semiconductors, for example, 3,4,9,10-perylenetetracarboxylic dianhydride (PTCDA) on surfaces such as mica, highly oriented pyrolytic graphite (HOPG), $\mathrm{KCl}(100)$, or $\mathrm{Au}(111){ }^{4-9}$ Growth and crystallization of these systems lead to a strong influence on the optical absorption and make DRS a highly universal method to measure adsorbates on surfaces. With this tool, a deeper understanding of the interaction, growth, ${ }^{7}$ charge transfer of molecules, ${ }^{10}$ as well as decoupling from solid surfaces ${ }^{8}$ has been obtained. Clarification of these effects is crucial for realization of true molecular electronics.

Besides the application to organic semiconductors on surfaces, also investigations of self-assembled monolayers

a)Electronic mail: fabian.nickel@fu-berlin.de
(SAM) of photochromic molecules by DRS have been carried out. ${ }^{11,12}$ There, the focus is not on the growth since these systems were prepared ex situ, but the important key property is the optical change upon illumination by various light sources. Photochromic molecules consist of two metastable states, which can be converted to each other by illumination with light of different wavelengths or changes in temperature. For material application, an immobilization of photochromic molecules on surfaces is essential. When in contact with a solid surface, switching properties are often found to be quenched and only partial or not fully reversible switching has been shown. Several species of different photoswitches have been investigated on surfaces, but so far no molecule in direct contact with a surface has been found that can be switched efficiently and reversibly by light only: For example, diarylethenes on HOPG and Bi(111) did not show reversibility after photoisomerization to a closed-form ${ }^{13}$ and a spiropyran containing a nitro group is even reversing its thermal stability on an $\mathrm{Au}(111)$ surface. ${ }^{14}$ On the other hand, systems using a bismuth substrate have been reported with promising results. Azobenzenes on $\mathrm{Bi}(111)$ can be switched to one state by $\mathrm{x}$ rays and to the other by thermal energy. ${ }^{15}$ Spiropyranes on $\mathrm{Bi}(110)$ were found in a photostationary state upon blue-light illumination, ${ }^{16}$ and spironaphthopyran on a Bi(111) surface can be reversibly switched by using a UV LED and thermal energy. ${ }^{17}$ From UV/Vis-spectroscopy in solution, the absorption bands of these molecules are already well known, but when in contact with a surface, the optical reflection represents a combination of the electronic and optical properties of the full system including the substrate. Therefore, DRS is a very suitable method to determine and quantify the ability to switch photochromic molecules on a surface. Explanation and characterization of the optical absorption even for molecules that do not show switching properties on the surface might lead to a deeper understanding of the switching process in proximity to the substrate.

The DRS setups for investigating organic semiconductors, ${ }^{5,9,18,19}$ SAMs ${ }^{11,12,20}$ or polymers ${ }^{21,22}$ have a typical 
white-light setup in common. A white-light source illuminates directly or via an optical fiber the sample surface, and the reflectivity is measured by a spectrometer. A typical advantage of this is the fast acquisition time and the flexibility. A huge drawback to measure photochromic molecules is their potential conversion by light absorption. In solution, quantum yields of photoswitchable molecules nearly reach $100 \%$, which means that upon measurement by white-light illumination a significant amount of molecules would be switched. In addition, the contrast within a spectrum is limited by the dynamic range of the spectrometer. A strong reflection in the visible part of spectra often leads to an increased noise in the UV or infrared range. Most photochromic molecules do not exhibit any absorption in the visible range in one of their states, which makes it necessary to have a good spectral quality over the whole UV/Vis range.

Here, we present an experimental setup that is capable of measuring photoisomerization of in-situ-deposited molecules in a UHV environment at variable temperature. It is designed to measure the change of the optical reflection and the switching kinetics during illumination. Using a monochromatic measurement beam in combination with photomultipliers (PMT) leads to a minimum light exposure of the molecules. In addition, this enables time-dependent measurements at single wavelengths such that the acquisition speed can be increased with less total light exposure. To maximize the signal-to-noise ratio (SNR), a reference beam can be used. Special effort was spent to optimize the setup in the UV range since, as mentioned before, photochromic molecules often do not exhibit absorption in the visible range. Therefore a dual-lamp with a deuterium and halogen source as well as UV-optimized optics is used. As a demonstration of the potential of our setup, measurements of the photochromic switch spironaphthooxazine (SNO) on the Bi(111) surface are presented. Besides the DRS of both isomerization states, the kinetics of the UV photoisomerization to the merocyanine state (MC) is quantified. By temperaturedependent measurements of the relaxation, activation barriers between the two metastable states are determined within an Arrhenius model.

\section{DIFFERENTIAL REFLECTANCE SPECTROSCOPY SETUP}

\section{A. Measurement principle}

Using the optical reflection of a surface-adsorbate system, the signal consists of contributions from the surface and adsorbate. Comparing the optical reflection of the hybrid system to the signal of the bare substrate enables one to extract information on the absorption of the adsorbate. This method allows for a very sensitive investigation of the change of the optical properties of the surface. The DRS signal is defined by the reflection $R(\lambda)$ at a certain wavelength $\lambda$ compared to the substrate reflection $R_{0}(\lambda)$ as

$$
\frac{\Delta R}{R}(\lambda)=\frac{R(\lambda)-R_{0}(\lambda)}{R_{0}(\lambda)} .
$$

To account for noise originating from the lamp and optical setup, a reference is introduced and the reflection is measured as

$$
R(\lambda)=\frac{I_{S}(\lambda)}{I_{R}(\lambda)},
$$

with the sample and reference PMT currents $I_{S}$ and $I_{R}$, respectively. Such a normalization has been already utilized in the literature and increases the signal-to-noise (SNR) ratio significantly. ${ }^{19}$

The change $\Delta R / R(\lambda)$ is not only originating from the optical absorption of the adsorbate but also from the change of optical constants of the surface. Therefore a direct inference to the properties of the adsorbing film is not feasible. By linearization of the Fresnel equations for the reflection of a layered system of vacuum, adsorbate, and semi-infinite substrate, an approximation for the complex dielectric functions of adsorbate and substrate can be given, ${ }^{23}$ but only for the case of highly transparent substrates, further simplifications to determine optical constants of adsorbates are possible. ${ }^{5} \mathrm{By}$ applying models or using the Kramers-Kronig relation, optical constants can also be determined for opaque substrates, e.g., gold or HOPG. ${ }^{24}$ Separation of the optical constants of molecule and substrate is not necessarily required for the investigation of photochromic systems, in particular, if the focus of interest lies on the kinetics of the switching process. Only a weak change of each optical constant is expected if there is no strong interaction between surface and switch. A detailed deconvolution into the contributions from the refractive index and extinction coefficient as described in the literature l,24,25 $^{1,2}$ is thus not in the scope of the experiment presented here. The important property to identify in these systems is the ability to switch reversibly and efficiently.

\section{B. UHV setup}

In surface science, entire sample handling under UHV conditions is necessary. Therefore, the presented DRS setup consists of a UHV chamber with a base pressure of $2 \times 10^{-10}$ mbar. A sample load-lock to quickly exchange substrates as well as a device to cleave surfaces (e.g., HOPG) is mounted on this chamber. For preparation of single-crystal surfaces, a sputter gun and heating stage are present. In Fig. 1(a), the main UHV body based on a CF100 T-piece is shown. Due to the small diameter $(100 \mathrm{~mm})$ of this chamber body, the distances between the sample and windows are reduced to only $62 \mathrm{~mm}$. This makes the use of in-vacuum lenses unnecessary. Six fused silica CF16 windows with an inner diameter of $16 \mathrm{~mm}$ are mounted and enable high optical transmission between 190 and $2500 \mathrm{~nm}$. The flanges of the two innermost windows on the horizontal axis are cut to reduce the angle for near-normal incidence to $11.5^{\circ}$ to the surface normal axis. The two outer windows on the horizontal axis offer the possibility for polarization-dependent measurements. The angle between these windows is $90^{\circ}$. The sample holder is directly mounted from the bottom of the CF100 T-piece and enables fast exchange and adjustment. The sample holder is shown in panel (b) of Fig. 1. For a stable operation of DRS, highest mechanical stability is required. When using a manipulator, mechanical oscillations or translations may occur. Therefore, a fixed sample holder was built. An ex situ manipulation of five axes (all except for azimuthal rotation) is available to adjust 


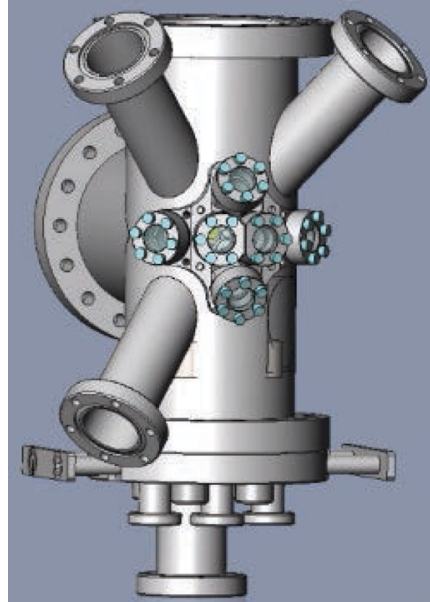

(a)

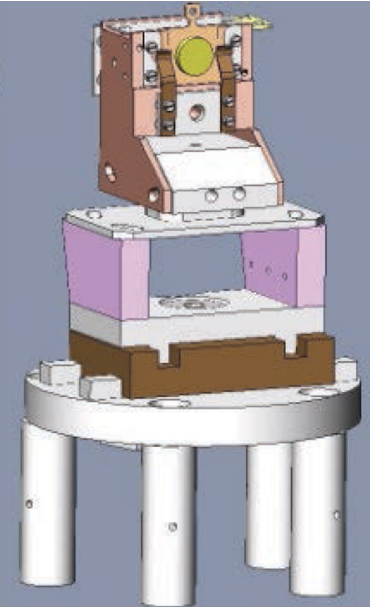

(b)
FIG. 1. (a) UHV chamber for in situ DRS measurements. The optical components are directly mounted on the CF16 windows in the center; three CF40 ports for evaporators are pointing toward the sample. (b) Sample holder designed for highest stability with the possibility of ex situ five-axis adjustment.

the optical setup. The sample is pressed strongly against an oxygen-free high thermal conductivity (OFHC) copper block by two spring bronze plates. A boron nitride heater plate is mounted on the top of this block for sample heating. For cooling, a liquid-nitrogen-cooled heat exchanger is connected to the main block by copper strands. The strands are required to decouple the sample holder from possible nitrogen boiling disturbances. Thermal insulation of the copper block is achieved by using two polyimide blocks as a connection to the $x-y$ manipulation of the socket. This leads to a very low thermal conductivity and a minimal temperature limited by thermal radiation. The temperature of the main block is measured by a LakeShore DT-670B-CO silicon diode. An accurate control of the temperature is achieved by PID-control of the heating power by a home-made LabView program. With that, stable temperatures with oscillations below $0.1 \mathrm{~K}$ were reached between 100 and $460 \mathrm{~K}$.

The three CF40 ports in Fig. 1(a) are pointing to the sample and enable mounting of different additional equipment with direct view on the sample. For investigations of photochromic molecules, a home-made Knudsen-cell evaporator with a quartz microbalance is mounted behind a valve.

\section{Optical setup}

Figure 2 presents a sketch of the optical components of the DRS setup. A nearly normal incidence has been chosen for the measurement of photochromic molecules on opaque substrates. In contrast to recent publications of similar setups, white-light illumination of the sample is not used. A double monochromator in combination with two photomultiplier tubes (PMTs) measures the reflectance.

A Bentham ILD-D2-QH lamp provides highly intense light from 200 to $300 \mathrm{~nm}$ with a low-noise deuterium $\left(\mathrm{D}_{2}\right)$ lamp and 300 to $2000 \mathrm{~nm}$ by a quartz halogen (QH) lamp. The illumination source is selected by a flip mirror (M1), which is controlled by the home-made measurement software.

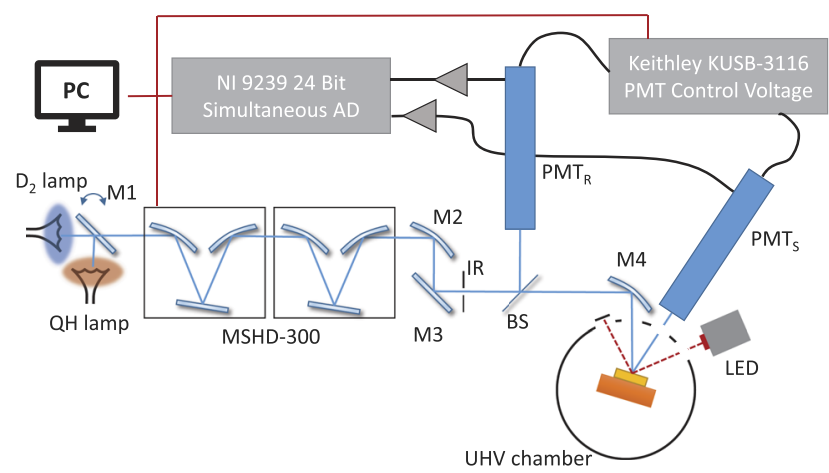

FIG. 2. Illustration of the optical setup: Light from a Deuterium/QuartzHalogen source (selection by flip-mirror M1) is monochromatized by a double monochromator. The beam is collimated by mirror M2. A fused silica beamsplitter (BS) redirects a fraction of the light to the reference $\mathrm{PMT}_{\mathrm{R}}$, whereas the mirror M4 focuses the beam into the UHV chamber. Detection of the reflected light from the sample is done by the sample PMT . Both PMT gains are controlled by a Keithley KUSB-3116, and the photocurrents are amplified by FEMTO amplifiers and subsequently digitized by a National Instruments $9239 \mathrm{AD}$ converter. In situ LED illumination is possible through various windows on the chamber.

The broadband light is monochromatized by a MSHD-300 double monochromator purchased from LOT-QuantumDesign $\mathrm{GmbH}$. A double monochromator has been chosen over a single one since the estimated change of reflection of submonolayers of molecules upon isomerization can be of the order of only $10^{-3}$. Stray light of a single monochromator can be of the same order of magnitude, which would lead to incorrect conclusions. Besides that, a low light exposure of the photochromic molecules is required to not interfere with the desired LED-illumination effect. A double monochromator minimizes the relative stray light down to $10^{-6}$. Three gratings are mounted in each of the monochromators to allow for broad range and detailed measurements in different regions of the spectrum from UV to NIR. For the spectra presented later on, a grating with $1200 \mathrm{l} / \mathrm{mm}$ and a blaze wavelength of $300 \mathrm{~nm}$ has been used, which provides sufficient intensity from 240 to $750 \mathrm{~nm}$. With a $24001 / \mathrm{mm}$ holographic $250 \mathrm{~nm}$ blaze grating, measurements down to $200 \mathrm{~nm}$ are possible, whereas for the infrared region a $1200 \mathrm{l} / \mathrm{mm}$ grating with a blaze of $750 \mathrm{~nm}$ is mounted. Additionally, filters blocking light below 350, 430, and $725 \mathrm{~nm}$ are introduced automatically between the two monochromators depending on the selected wavelength to suppress light of higher orders.

The slits of the monochromator can be adjusted between 0.05 and $8 \mathrm{~mm}$ width, where theoretical bandwidths between 0.07 and $11 \mathrm{~nm}$ are reached. Behind the exit slit of the double monochromator, an f/4 focal length focussing mirror (M2) is placed. The collimated beam is directed by mirror M3 to an iris aperture (IR), to limit the beam diameter. Uncoated fused silica is used as a beamsplitter. This beamsplitter has about $4 \%$ reflectance to split a reference beam into the reference photomultiplier $\mathrm{PMT}_{\mathrm{R}}$. The sample beam is focused again with a UV-enhanced off-axis parabolic mirror (M4) with a focal length of $15.2 \mathrm{~mm}$. Therefore, the focus is behind the sample, enabling for a smaller spot on the exit window than on the entrance window. This is intended to reduce the eventuality of 
clipping of the beam. Such a clipping would lead to a vulnerability to increased noise. The whole beam path is covered by a tube system and black-anodized aluminum foil to block all ambient light from the experiment.

Photomultipliers from Hamamatsu of the type H10426-01 are used. Their advantage is a rather broad spectral sensitivity $(185-850 \mathrm{~nm})$ and a very large sensitive area with a diameter of $25 \mathrm{~mm}$. This enables detection of the whole reflected beam when mounted behind the CF16 vacuum window (diameter $16 \mathrm{~mm}$ ). Typical spectrometers as used in white-light setups employ CCD chips to measure DRS. In Ref. 19, an NMOStype Si diode array (Hamamatsu S3904) is used, which has a spectral response between 0.05 and $0.25 \mathrm{~A} / \mathrm{W}$. Compared to this, the Hamamatsu PMTs exhibit a sensitivity in the range between 10 and $70 \mathrm{~mA} / \mathrm{W}$. Together with a gain of, e.g., $10^{5}$ (the gain can be controlled between $10^{3}$ and $10^{7}$ ), the PMT is around four orders of magnitude more sensitive at a wavelength of $600 \mathrm{~nm}$. A further advantage compared to a white-light setup is the reduced exposure to light during the measurement. Assuming a sample consisting of photochromic switches that are switching equally effective as in solution (cross sections around $10^{-17} \mathrm{~cm}^{2}$ ), ${ }^{26}$ conventional DRS whitelight setups would lead to a switching of the molecules with time constants in the range of several $100 \mathrm{~s}$. This would interfere with typical measurement times for the different isomerization states of the molecule-surface systems. In contrast to that, our setup allows measurement times of several days since the light flux is orders of magnitude below that needed for CCD-based detectors.

Both PMTs are equipped with computer-controlled shutters (Thorlabs SH1/M for the sample and SHB1 for the reference). The shutters are operated by two different safety interlocks, to close either when the current exceeds a certain limit or when an LED for sample illumination is operated. Hereby, the sample illumination is possible by different LEDs, mounted on CF16 windows. Typically, two different LEDs are mounted for experiments with photochromic molecules to allow for possible bi-directional light-induced switching. Additional optics can be used to increase the intensity further by focussing to a smaller area.

Several variations of the optical setup can be imagined. For investigations of the optical anisotropy, the outer windows can be used for DRS. The polarization dependency can be analyzed by a Glan-Thompson polarizing prism as, for example, described by Navarro-Quezada et al. ${ }^{27}$

\section{Acquisition and software}

The data acquisition and control are done by a National Instruments (NI) 9239 analog-digital converter and Keithley KUSB-3116 digital-analog converter, respectively. A very stable control voltage for the Hamamatsu PMT is required for an accurate internal high voltage generation. This control voltage is supplied by the Keithley device, which was found to deliver the most stable operation over long times. Any instability of the 0.4-1.4 V control voltage directly influences the measurement. The detection of the signals is realized by amplifying the currents in the order of microamperes with a FEMTO DDPCA-300 current amplifier for each PMT and then logged to the personal computer (PC) by the NI 9239 converter. Especially in the case of light sources with a high frequency noise, such as xenon arc lamps, a fast and simultaneous signal acquisition of the reference and sample is necessary. To account for this, NI 9239 uses independent AD converters, which are triggered simultaneously and convert with an accuracy of up to 24 bit and $50 \mathrm{kS} / \mathrm{s}$.

Automation is of high importance for the measurement of photochromic systems since typically cross sections for photoswitching on surfaces are reduced by several orders of magnitude ${ }^{13,17}$ and result in extensive measurement times. To determine suitable combinations of molecules and substrates, experiments in a reproducible and reliable manner are required. As mentioned before, the temperature control is automatized, which enables recording of temperature ramps and systematic studies. Measurements are executed and controlled by a home-made LabView program. A spectrum can hereby be split into parts with different PMT control voltages, therefore increasing the dynamical range of a spectrum by orders of magnitude. For the sake of fast acquisition, presented spectra are taken with an integration time of $700 \mathrm{~ms}$ per point and a step width of $2 \mathrm{~nm}$. For the demonstrated application, besides taking full spectra, also other types of measurements are necessary. Measuring the kinetics of switching processes is possible by only recording certain points of a spectrum to increase the speed of measurement. For a time-dependent measurement without additional illumination, either only a certain wavelength can be recorded or different wavelengths alternatingly. For a highly accurate and drift-resistant investigation of switching kinetics, a point on an absorption peak and one on a more or less constant area can be measured. Rates can be determined by taking the asymmetry of an absorption peak by

$$
\operatorname{asym}\left(\lambda_{1}, \lambda_{2}\right)=\frac{R\left(\lambda_{1}\right)-R\left(\lambda_{2}\right)}{R\left(\lambda_{1}\right)+R\left(\lambda_{2}\right)} .
$$

In that way, a time-dependent measurement of temperature or light-induced switching is possible with an accurate determination of rate constants. By taking full spectra before and after the switching series, the asymmetry can be related to the amount of switched molecules.

Since the use of additional LED illumination is not possible while scanning with the PMTs, the shutters of the PMTs need to be closed and their high voltage shut down for intense LED illumination. Therefore, recording the asymmetry of an absorption peak and light-exposure are done alternatingly.

\section{E. Noise and drift}

For a fast acquisition, the noise needs to be minimized. Typical noise deviations in a white-light setup of the $\Delta R / R$ signal due to instability of lamps without using a reference signal were often found to be in the order of $10^{-2}$, while with reference this could be around $3 \times 10^{-4}$ for long integration times of $6 \mathrm{~s}{ }^{19}$

Figure 3 presents time-dependent recordings of the reflection of a clean $\mathrm{Au}(111)$ substrate and reference at a wavelength of $500 \mathrm{~nm}$. Each point has been taken with an integration time of $1 \mathrm{~s}$, and the points are normalized to the first point before subtracting a value of 1 . The red dots represent the signal of the sample PMT; the black dots represent that of the reference 


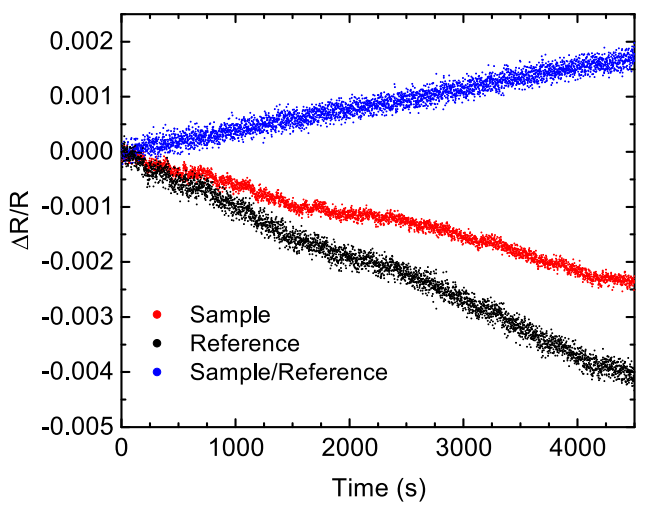

FIG. 3. Time-dependent measurement of the photocurrents of both PMTs (red dots for the sample and black for the reference) and normalized to the first data point for each PMT. A clean Au(111) substrate was used, and the reflection was measured at $\lambda=500 \mathrm{~nm}$ at room temperature.

PMT, and the blue dots represent that of the sample/reference normalized signal, respectively. Photocurrents are $2.5 \mu \mathrm{A}$ for the sample PMT and 2.1 $\mu \mathrm{A}$ for the reference PMT, respectively. The temperature was left at room temperature. Different drifts are apparent in the measurement. The overall nearly linear drift to smaller reflected intensity for both PMTs is presumably originating from a combination of light-source and gain drift.

The standard deviation, calculated from the data of Fig. 3 by subtracting straight lines for each series, is $7 \times 10^{-5}$ for the sample PMT, $1 \times 10^{-4}$ for the reference PMT, and thus $1 \times 10^{-4}$ as well for the normalized signal. Utilizing PMTs for recording DRS therefore leads to a reduced noise level in our setup. Compared to the noise level reported in Ref. 19, it is improved by at least a factor of three for the normalized signal using a reference. Without reference, the improvement is even higher, more than two orders of magnitude. ${ }^{19}$ A noise level better than $10^{-4}$ is crucial for the measurements. The noise is dominated by the shot noise of the PMT. Using the photocurrents, gains ( $10^{4}$ for sample, $2 \times 10^{5}$ for reference), and integration time a shot noise of $4 \times 10^{-5}$ for the sample PMT and $1 \times 10^{-4}$ for the reference PMT is calculated, respectively. ${ }^{28}$ Besides the linear drift, shorter drifts are visible around $1700 \mathrm{~s}$ at both PMTs, most likely originating from a drift of the light source. Instabilities of the light source and other influences such as vibrations of the monochromator can efficiently be separated by normalizing to the reference signal, hence leading to a stable measurement. Remaining long-term drifts over time spans of hours can be corrected by later data treatment as explained in the context of the application.

\section{APPLICATION TO PHOTOCHROMIC MOLECULES}

\section{A. Experiment with spironaphthooxazine}

As a demonstration of the various possibilities for application of the described setup, results of the photochromic spironaphthooxazine (SNO, full chemical name 1,3,3-trimethylindolinonaphthospirooxazine) on a $\mathrm{Bi}(111)$ surface will be shown. SNO is shown in Fig. 4 and can be converted by UV-light illumination to merocyanine (MC). A conversion backwards to SNO is possible in solution by

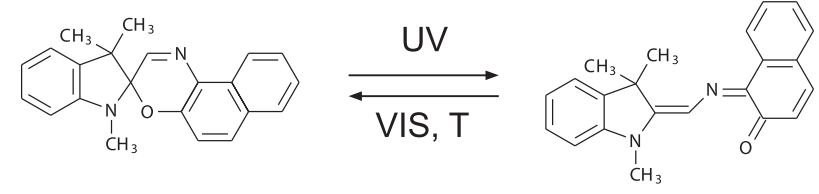

FIG. 4. Scheme of the molecular switch spironaphthooxazine (SNO), which can be converted in solution by UV illumination to merocyanine (MC) and reversibly back by either visible-light illumination or increase of temperature.

either visible-light illumination (often red) or a temperature increase.

The Bi(111) surface was prepared by standard sputter and annealing cycles. For sputtering, $600 \mathrm{eV} \mathrm{Ar}^{+}$ions were used, and for annealing, the crystal was kept for 15 min slightly above $350 \mathrm{~K}$. The base pressure of the UHV chamber was $2 \times 10^{-10}$ mbar. SNO molecules, purchased from TCI Chemicals, were evaporated from a home-built evaporator with a Knudsen cell at a temperature of about $363 \mathrm{~K}$ with a pressure of $5 \times 10^{-9}$ mbar. The substrate temperature was kept at $200 \mathrm{~K}$ for evaporation, to increase the rate of adsorption on the surface. Prior to the measurements, all optical components have been warmed up for several hours to reduce drifts of the signal. The settings were adjusted for an optimized acquisition speed. Hereby, $700 \mathrm{~ms}$ integration time per point and $2 \mathrm{~nm}$ step width were used. The slits were set to $1 \mathrm{~mm}$ with a theoretical bandwidth of $1.35 \mathrm{~nm}$. A measurement spot of $3 \times 7 \mathrm{~mm}^{2}$ on the Bi(111) was used.

\section{B. Adsorption on $\mathrm{Bi}(111)$}

Figure 5 shows the measurement of adsorbed SNO on $\mathrm{Bi}(111)$. It is important to care about correction and thickness calibration of the DRS in detail. Panel 5(a) shows the DRS of the freshly adsorbed SNO on $\mathrm{Bi}(111)$ of the pristine sample (black line) and after 5:30 h of experiments (red line) without normalization to the reference PMT. By the alreadydescribed drift of the DRS, the signal changes by 0.01 . Beside that, noise in the range of $1 \times 10^{-3}$ is visible as well as a spike from the change of the illumination source at $300 \mathrm{~nm}$. Spikes and remaining noise originate from instabilities of the optical setup, e.g., grating vibrations or inaccuracies. In panel (b), the reference PMT has been used for normalization and in addition a simple correction for the drift is applied. For the latter, a sensitivity drift was calculated, which is defined as the factor between the photocurrents for each PMT in the puresubstrate spectrum and the one in the recorded spectrum at a certain wavelength. A wavelength of $720 \mathrm{~nm}$ is chosen for this correction since there is no absorption by the adsorbate expected and a good signal can be acquired. The calculated factor is applied to the whole spectrum. This drift could originate from changes in the PMTs or drift of the control voltage for the internal high voltage generation of the PMTs. Applying this factor leads to well-matching spectra, even over long time spans. By using the reference signal, the noise is strongly reduced without further adjustments. The drift of the PMTs, as shown in Sec. II E, dominates the results.

In contrast to the low-temperature measurements, molecules do not permanently stick after completion of the first layer on a $\mathrm{Bi}(111)$ surface. Therefore, to determine the 
(a)

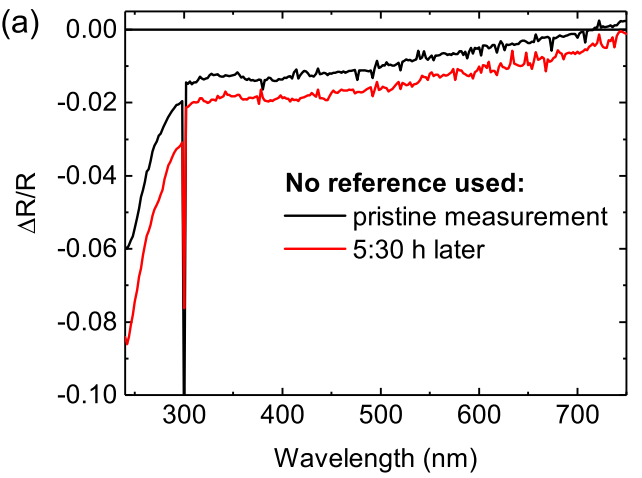

(b)

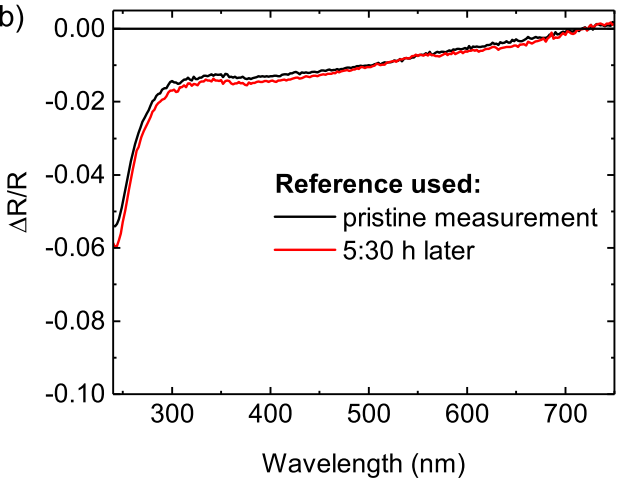

FIG. 5. DRS of SNO on Bi(111) (a) without using the reference directly after evaporation (black line) and after 5:30 h (red line). (b) Exactly the same measurements including the use of the reference beam and correcting for a gain drift by referencing to the $720 \mathrm{~nm}$ intensity. Measurements were taken at $T=200 \mathrm{~K}$.

amount of molecules on the surface, measurements at room temperature were carried out. For the similar molecule spironaphthopyran on the same surface, this has been done before by x-ray absorption spectroscopy. ${ }^{17}$ Figure 6 shows the thickness calibration for $\mathrm{SNO}$ on $\mathrm{Bi}(111)$. A quartz microbalance mounted in the evaporator provides a measure proportional to the amount of molecules arriving at the sample surface during evaporation. The quartz is cooled by ice water to improve sticking of the molecules. Since the adsorbate does not form a permanent second layer on $\mathrm{Bi}(111)$ at room temperature, the coverage saturates exponentially. To measure and fit this saturation, different steps of evaporation were carried out successively with recording DRS in between. Panel (a) of Fig. 6 shows the corresponding DRS spectra, whereas panel (b) plots the intensity at $245 \mathrm{~nm}$ for each evaporation step. The fit leads to a saturation of the DRS signal at -0.061 for one monolayer (ML). Due to inaccuracies of this method and drifts of the setup, such a calibration has an estimated error of around $10 \%$. The following experiments were carried out with a $245-\mathrm{nm}$ signal of around -0.055 which corresponds to a coverage of 0.9(1) ML.

\section{UV-induced switching}

To demonstrate the ability of the DRS setup for detecting the photochromism of molecules on a surface, a submonolayer of SNO on $\mathrm{Bi}(111)$ was illuminated by a UV LED with a wavelength of $365 \mathrm{~nm}$ and a photon flux density of $\phi=2.8(5)$ $\times 10^{15}$ photons s$^{-1} \mathrm{~mm}^{-2}$ at the surface (measured by means of
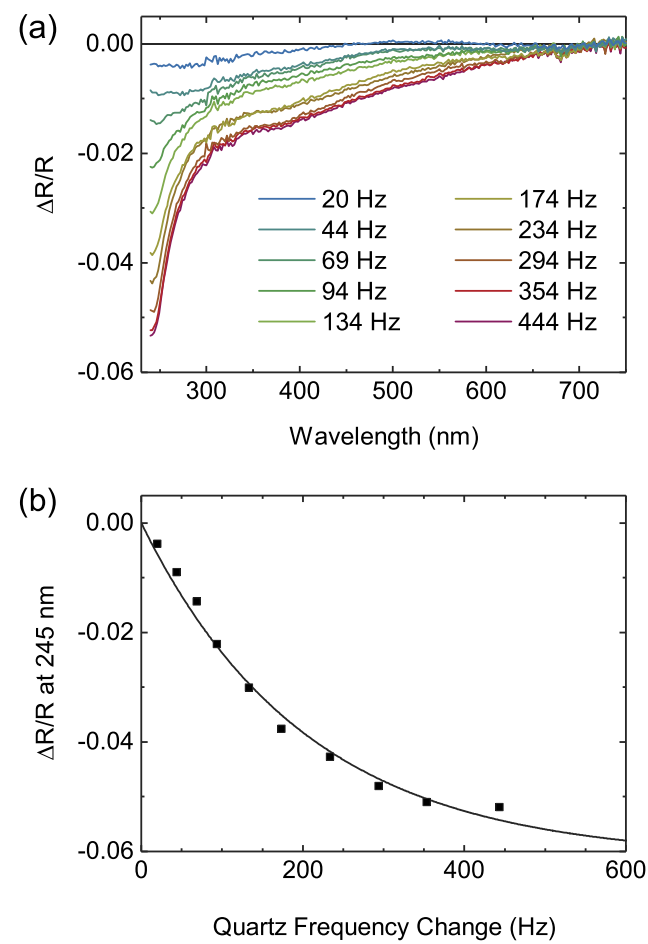

FIG. 6. Stepwise evaporation of SNO on Bi(111) with DRS measurements after each step. (a) DRS spectra for different coverages. The frequency change of the quartz microbalance serves as a measure of the evaporated amount of molecules. (b) Signal at $245 \mathrm{~nm}$ as a function of the microbalance frequency change.

a power meter). The illumination spot has approximately a size of $9 \times 9 \mathrm{~mm}^{2}$ with a rectangular shape and is therefore larger than the measurement spot of the DRS setup. Experiments were carried out at a temperature of $200 \mathrm{~K}$ with a coverage slightly below one ML (cf. Sec. III B). With this illumination, the molecules can be very quickly switched in solution from SNO to MC because of a quantum yield of up to $0.41 .{ }^{26}$ Due to the high sensitivity of the DRS, evaluation of the changes for short LED illumination times is possible.

Figure 7 shows in panel (a) the total change between the DRS after evaporation (black line) and after $94 \mathrm{~s}$ of accumulated UV LED illumination (red line). In panel (b), the asymmetry, as defined in Eq. (3), between the intensity at 240 and $290 \mathrm{~nm}$ is plotted for each illumination step. The change in DRS between the pristine and the UV-illuminated sample can be compared to UV/Vis spectra of the SNO and $\mathrm{MC}$ form in solution. Spiropyranes and SNO have been investigated intensely. Their absorption spectra reveal for SNO nearly no absorption in the visible part of the spectrum and for MC a peak around $600 \mathrm{~nm} \cdot{ }^{26,29}$ This $600-\mathrm{nm}$ peak has often a double-peak structure, depending on the configuration of the carbon bonds. ${ }^{29}$ We can identify a very similar change of the DRS spectrum, even though here the signal is a combination of substrate and adsorbate properties. The intensity of the reflectance in the UV region increases, whereas there is more absorption around a maximum at $625 \mathrm{~nm}$. Knowing the change of absorption of the adsorbate/substrate system is highly important to identify candidates and wavelengths for the reversible switching. Reverse photoisomerization has not yet been achieved for this system. With deeper knowledge through 
(a)
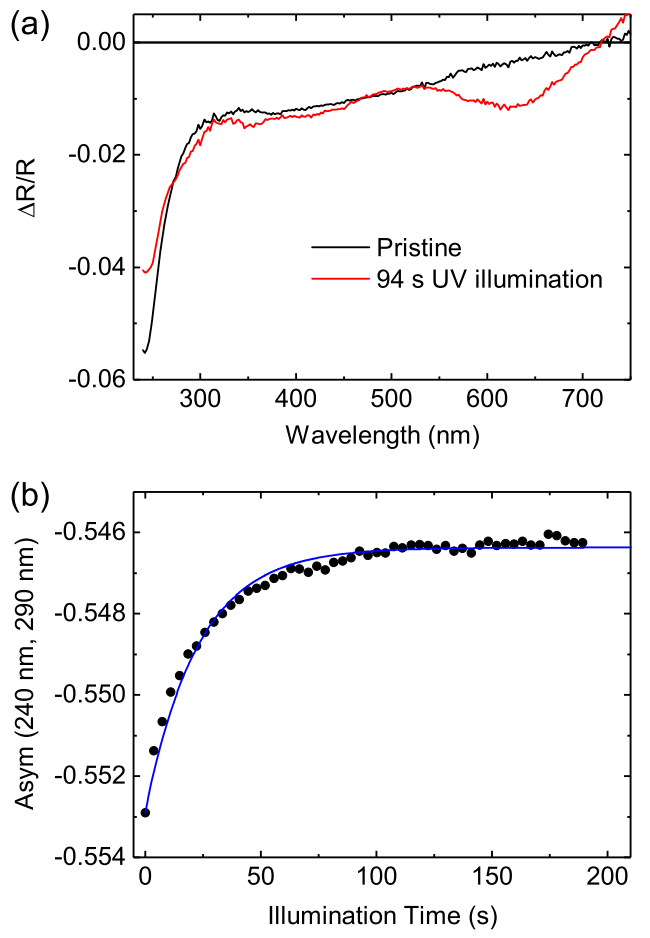

FIG. 7. (a) $\mathrm{SNO}$ on $\mathrm{Bi}(111)$ in the pristine state and after $94 \mathrm{~s}$ UV illumination. (b) Time dependence of the effect: stepwise illumination for $3.7 \mathrm{~s}$ and recording of the asymmetry of the 240 and $290 \mathrm{~nm}$ reflectance.

comparison of different photochromic molecules on surfaces, it will be possible to tailor systems to reach this important milestone.

The rate for the SNO-to-MC conversion can be measured by the DRS setup as well. To do so, the pristine SNO has been subjected to 3.7-s illumination steps and alternating recordings of the asymmetry. The advantage of this method is that the measurement of the clean substrate is not taken into account, other than for the DRS. Therefore it is independent of long-term drifts, but the asymmetry cannot be directly related to DRS intensity. An exponential function with a time constant of 24(1) s fits to the measurement, as presented in Fig. 7(b). Hereby, the first point without illumination has been determined with higher accuracy and kept fixed for the fit, resulting in a systematic deviation from a singleexponential function. The origin of this effect remains unclear but may be attributed to a more complex behavior of the switching kinetics, e.g., different adsorption sites or cooperative effects.

By the measured photon flux density, the effective cross section can be calculated as $\sigma_{\text {eff }}=(\phi \tau)^{-1}=1.4(3) \times 10^{-19} \mathrm{~cm}^{2}$. Compared to literature values for cross sections of photochromic switching on surfaces, this is a highly effective process. In solutions, typically cross sections orders of magnitude higher are measured. ${ }^{30,31}$ Hence there is still a high potential to increase the switching efficiency while in contact with a surface. So far, in direct contact with surfaces, cross sections that are by orders of magnitude lower than those for experiments in solutions have been observed. The spironaphthopyranto-MC conversion on $\mathrm{Bi}(111)$ exhibited a cross section in the order of $10^{-20} \mathrm{~cm}^{2}$ for elevated temperatures and even below $10^{-21} \mathrm{~cm}^{2}$ at $200 \mathrm{~K} .{ }^{17}$ Other photochromic molecules were measured with even lower effective cross sections such as $10^{-23} \mathrm{~cm}^{2}$ for azobenzenes in thin films on the $\mathrm{Bi}(111)$ surface. $^{32}$

Switching back by a red-light source was not possible for this system, but by heating the substrate to higher temperatures such as $250 \mathrm{~K}$, the molecules relax back to the $\mathrm{SNO}$ form.

\section{Determination of the activation energy}

The temperature relaxation at slightly elevated temperatures can be used to identify the heights of the energy barriers in a simple Arrhenius model. The energetics of molecules on surfaces can be drastically different compared to those in solution. For example, a nitro-containing spiropyran was found to be stabilized in the open merocyanine form on the $\mathrm{Au}(111)$ surface. ${ }^{14}$ Investigating the energy barriers involved in the switching process and comparing them to their solution values lead to the possibility of tailoring the properties on the surface. We determine here the energy barrier leading to the temperature-dependent relaxation of MC to SNO.

Figure 8 shows the result of temperature-dependent relaxation measurements. Panel (a) presents relaxation curves of the 240-to-290-nm asymmetry as a function of time for five different temperatures from 240 to $260 \mathrm{~K}$. The corresponding logarithms of the rate over $1 / \mathrm{T}$ are shown in panel (b). In an Arrhenius model with an energy barrier $E_{A}$, the rate is $k(T)=A \times \mathrm{e}^{-E_{A} /(R T)}$, with $R$ being the gas constant and $A$ being a pre-exponential factor depending on the vibrational freedom, which is in the order of $10^{9}-10^{14} \mathrm{~s}^{-1}$ for measurements in solutions. ${ }^{26,33}$ Each relaxation series has been fitted with a single exponential function but different zero lines to determine the switching rate. Due to the different temperature, changes of
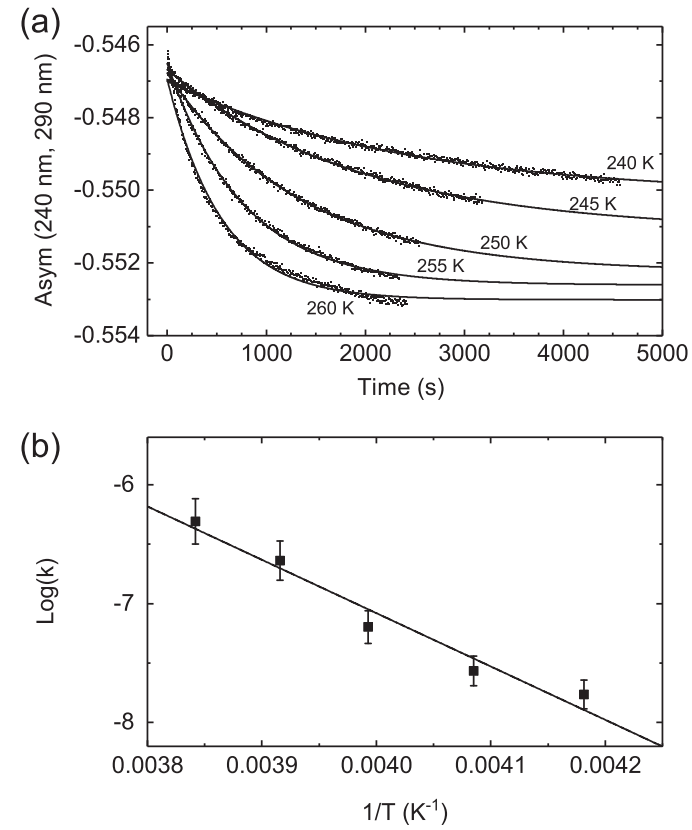

FIG. 8. Measurement of thermal relaxation of SNO on Bi(111). (a) Time dependence of the asymmetry at different temperatures and their fits by single exponential functions (solid lines) and (b) evaluation of the rates by a simple Arrhenius behavior and a fit to determine the energy barrier (solid line). 
the substrate reflectance are likely and lead to a different magnitude of the observed effect. The fits reproduce the data well, with a small deviation for the highest temperature at $260 \mathrm{~K}$. From the data, it is not clear whether a drift has an impact on the fit or an additional relaxation channel is available at higher temperatures. Fitting the data in panel (b) to the Arrhenius behavior results in $E_{A}=37(4) \mathrm{kJ} \mathrm{mol}^{-1}$ and $A=5(1) \times 10^{5} \mathrm{~s}^{-1}$. For this molecule in different solutions, barriers between 61 and $78 \mathrm{~kJ} \mathrm{~mol}^{-1}$ have been found. ${ }^{26,33}$ The difference can be explained with a strong influence of the substrate on the switching process. The deviation from the fit in Fig. 8(b) hints toward a more complex behavior than a simple Arrheniuslike energy barrier. It is remarkable that the prefactor is much lower compared to typical values found in solution. This could be explained by the reduced freedom of the molecule on the surface but could also originate from a deficiency of the model. Nevertheless, the variation of temperature allows for a deeper analysis of the switching kinetics. Additional measurements of the kinetics for UV illumination at different temperatures might lead to a determination of the barriers in a more advanced energy scheme, as has been done in Ref. 17 by using x-ray absorption spectroscopy.

\section{CONCLUSION}

A DRS setup suitable for the investigation of photochromic molecules on solid surfaces at low coverages has been presented. Hereby, monochromatic light is used and the reflectance is detected by PMTs, in order to keep the light exposure of the molecules at a minimum. The stable mechanical setup and PID-controlled temperature enable reliable measurements between 100 and $460 \mathrm{~K}$. A low noise of the spectra and measurement of switching kinetics allow systematic investigations of photochromic molecules on opaque substrates. As a demonstration, a monolayer of spironaphthooxazine molecules on a Bi(111) surface has been measured. The measurement of the highly efficient conversion to merocyanine is shown together with an evaluation of the activation energy for the thermal back-relaxation.

\section{ACKNOWLEDGMENTS}

Financial support from DFG through Sfb 658 is gratefully acknowledged. The authors thank Lukas Kemmler and Janina Drauschke for their assistance during first experiments with the presented setup. Ivar Kumberg is acknowledged for proofreading of the manuscript. The precision mechanics workshop of the Department of Physics of the Freie Universität Berlin is acknowledged for manufacturing the UHV chamber and most of the vacuum parts.

${ }^{1}$ R. Forker, M. Gruenewald, and T. Fritz, "Optical differential reflectance spectroscopy on thin molecular films," Annu. Rep. Sect. C: Phys. Chem. 108, 34-68 (2012).

${ }^{2}$ R. E. Hummel, "Differential reflectometry and its application to the study of alloys, ordering, corrosion, and surface properties," Phys. Status Solidi A 76, 11-44 (1983).

${ }^{3}$ D. E. Aspnes and A. A. Studna, "Anisotropies in the above-band-gap optical spectra of cubic semiconductors," Phys. Rev. Lett. 54, 1956-1959 (1985).
${ }^{4}$ H. Proehl, T. Dienel, R. Nitsche, and T. Fritz, "Formation of solid-state excitons in ultrathin crystalline films of PTCDA: From single molecules to molecular stacks," Phys. Rev. Lett. 93, 097403 (2004).

${ }^{5}$ H. Proehl, R. Nitsche, T. Dienel, K. Leo, and T. Fritz, "In situ differential reflectance spectroscopy of thin crystalline films of PTCDA on different substrates," Phys. Rev. B 71, 165207 (2005).

${ }^{6}$ R. Forker, T. Dienel, T. Fritz, and K. Müllen, "Optical evidence for substrateinduced growth of ultrathin hexa-peri-hexabenzocoronene films on highly oriented pyrolytic graphite," Phys. Rev. B 74, 165410 (2006).

${ }^{7}$ T. Dienel, C. Loppacher, S. C. B. Mannsfeld, R. Forker, and T. Fritz, "Growth-mode-induced narrowing of optical spectra of an organic adlayer," Adv. Mater. 20, 959-963 (2008).

${ }^{8}$ R. Forker, D. Kasemann, T. Dienel, C. Wagner, R. Franke, K. Müllen, and T. Fritz, "Electronic decoupling of aromatic molecules from a metal by an atomically thin organic spacer," Adv. Mater. 20, 4450-4454 (2008).

${ }^{9}$ R. Forker and T. Fritz, "Optical differential reflectance spectroscopy of ultrathin epitaxial organic films," Phys. Chem. Chem. Phys. 11, 2142-2155 (2009).

${ }^{10}$ A. Baby, M. Gruenewald, C. Zwick, F. Otto, R. Forker, G. van Straaten, M. Franke, B. Stadtmüller, C. Kumpf, G. P. Brivio, G. Fratesi, T. Fritz, and E. Zojer, "Fully atomistic understanding of the electronic and optical properties of a prototypical doped charge-transfer interface," ACS Nano 11, 10495-10508 (2017).

${ }^{11}$ T. Moldt, D. Brete, D. Przyrembel, S. Das, J. R. Goldman, P. K. Kundu, C. Gahl, R. Klajn, and M. Weinelt, "Tailoring the properties of surfaceimmobilized azobenzenes by monolayer dilution and surface curvature," Langmuir 31, 1048-1057 (2015).

${ }^{12}$ T. Moldt, D. Przyrembel, M. Schulze, W. Bronsch, L. Boie, D. Brete, C. Gahl, R. Klajn, P. Tegeder, and M. Weinelt, "Differing isomerization kinetics of azobenzene-functionalized self-assembled monolayers in ambient air and in vacuum," Langmuir 32, 10795-10801 (2016).

${ }^{13}$ F. Nickel, M. Bernien, M. Herder, S. Wrzalek, P. Chittas, K. Kraffert, L. M. Arruda, L. Kipgen, D. Krüger, S. Hecht, and W. Kuch, "Light-induced photoisomerization of a diarylethene molecular switch on solid surfaces," J. Phys.: Condens. Matter 29, 374001 (2017).

${ }^{14}$ M. Piantek, G. Schulze, M. Koch, K. J. Franke, F. Leyssner, A. Krüger, C. Navío, J. Miguel, M. Bernien, M. Wolf, W. Kuch, P. Tegeder, and J. I. Pascual, "Reversing the thermal stability of a molecular switch on a gold surface: Ring-opening reaction of nitrospiropyran," J. Am. Chem. Soc. 131, 12729-12735 (2009).

${ }^{15}$ A. Krüger, M. Bernien, C. F. Hermanns, and W. Kuch, "X-ray-induced reversible switching of an azobenzene derivative adsorbed on $\mathrm{Bi}(111)$," J. Phys. Chem. C 118, 12916-12922 (2014).

${ }^{16}$ G. Schulze, K. J. Franke, and J. I. Pascual, "Induction of a photostationary ring-opening-ring-closing state of spiropyran monolayers on the semimetallic Bi(110) surface,” Phys. Rev. Lett. 109, 026102 (2012).

${ }^{17}$ F. Nickel, M. Bernien, K. Kraffert, D. Krüger, L. M. Arruda, L. Kipgen, and W. Kuch, "Reversible switching of spiropyran molecules in direct contact with a Bi(111) single crystal surface," Adv. Funct. Mater. 27, 1702280 (2017).

${ }^{18}$ U. Heinemeyer, K. Broch, A. Hinderhofer, M. Kytka, R. Scholz, A. Gerlach, and F. Schreiber, "Real-time changes in the optical spectrum of organic semiconducting films and their thickness regimes during growth," Phys. Rev. Lett. 104, 257401 (2010).

${ }^{19}$ H. Zaglmayr, C. G. Hu, L. D. Sun, and P. Zeppenfeld, "Optical referencing in differential reflectance spectroscopy,” Meas. Sci. Technol. 25, 115603 (2014).

${ }^{20}$ C. Weber, L. Pithan, A. Zykov, S. Bommel, F. Carla, R. Felici, C. Knie, D. Bléger, and S. Kowarik, "Multiple timescales in the photoswitching kinetics of crystalline thin films of azobenzene-trimers," J. Phys.: Condens. Matter 29, 434001 (2017).

${ }^{21}$ C. Weber, T. Liebig, M. Gensler, L. Pithan, S. Bommel, D. Bléger, J. P. Rabe, S. Hecht, and S. Kowarik, "Light-controlled 'molecular zippers' based on azobenzene main chain polymers," Macromolecules 48, 1531-1537 (2015).

${ }^{22}$ C. Weber, T. Liebig, M. Gensler, A. Zykov, L. Pithan, J. P. Rabe, S. Hecht, D. Bléger, and S. Kowarik, "Cooperative switching in nanofibers of azobenzene oligomers," Sci. Rep. 6, 25605 (2016).

${ }^{23}$ J. D. E. McIntyre and D. E. Aspnes, "Differential reflection spectroscopy of very thin surface films," Surf. Sci. 24, 417-434 (1971).

${ }^{24}$ R. Nitsche and T. Fritz, "Determination of model-free Kramers-Kronig consistent optical constants of thin absorbing films from just one spectral 
measurement: Application to organic semiconductors," Phys. Rev. B 70, 195432 (2004).

${ }^{25}$ A. B. Djurišić, T. Fritz, and K. Leo, "Modeling the optical constants of organic thin films: Application to 3,4,9,10-perylenetetracarboxylic dianhydride (PTCDA)," Opt. Commun. 183, 123-132 (2000).

${ }^{26}$ A. K. Chibisov and H. Görner, "Photoprocesses in spirooxazines and their merocyanines,” J. Phys. Chem. A 103, 5211-5216 (1999).

${ }^{27}$ A. Navarro-Quezada, M. Aiglinger, E. Ghanbari, T. Wagner, and P. Zeppenfeld, "Polarization-dependent differential reflectance spectroscopy for real-time monitoring of organic thin film growth," Rev. Sci. Instrum. 86, 113108 (2015).

${ }^{28}$ Hamamatsu, Photomultiplier Tubes: Basics and Applications, 3rd ed. (Hamamatsu Photonics K.K., Hamamatsu, 2007).

${ }^{29}$ F. Maurel, J. Aubard, A. Samat, M. Rajzmann, and R. Guglielmetti, "Interplay between theory and experiment in organic photochromism: Example of spirooxazine ring opening/closing equilibrium,” Mol. Cryst. Liq. Cryst. 430, 221-226 (2005).

${ }^{30}$ A. K. Chibisov and H. Görner, "Photochromism of spirobenzopyranindolines and spironaphthopyranindolines," Phys. Chem. Chem. Phys. 3, 424-431 (2001)

${ }^{31}$ H. Görner, "Photochromism of nitrospiropyrans: Effects of structure, solvent and temperature," Phys. Chem. Chem. Phys. 3, 416-423 (2001).

${ }^{32} \mathrm{C}$. Bronner and $\mathrm{P}$. Tegeder, "Photo-induced and thermal reactions in thin films of an azobenzene derivative on Bi(111)," New J. Phys. 16, 053004 (2014).

${ }^{33}$ E. Pottier, R. Dubest, R. Guglielmetti, P. Tardieu, A. Kellmann, F. Tfibel, P. Levoir, and J. Aubard, "Effets de substituant, d'hétéroatome et de solvant sur les cinétiques de décoloration thermique et les spectres d'absorption de photomérocyanines en série spiro[indoline-oxazine]," Helv. Chim. Acta 73, 303-315 (1990). 